WALter LitTMan (Minneapolis, MN)

Stephen TAYlor (Auckland)

\title{
THE BALAYAGE METHOD: BOUNDARY CONTROL OF A THERMO-ELASTIC PLATE
}

Abstract. We discuss the null boundary controllability of a linear thermoelastic plate. The method employs a smoothing property of the system of PDEs which allows the boundary controls to be calculated directly by solving two Cauchy problems.

1. Introduction. In this paper we apply a method of boundary controllability to the system

$$
\begin{array}{lll}
(1.1) & w_{t t}=-\Delta^{2} w-\alpha \Delta \theta, \theta_{t}=\beta \Delta \theta+\alpha \Delta w_{t}-\gamma \theta & \text { in } \Omega \times(0, T], \\
(1.2) & w(x, 0)=w_{0}(x), w_{t}(x, 0)=w_{1}(x), \theta(x, 0)=\theta_{0}, & x \in \Omega, \\
(1.3) & B_{i}(w, \theta)=g_{i}, \quad i=1,2,3, & \text { on } \partial \Omega \times(0, T],
\end{array}
$$

where $\Omega \subset \mathbb{R}^{2}$ is a bounded domain, $\alpha>0, \beta>0, \gamma \geq 0$ and $T>0$ are constants, $B_{i}$ are appropriate boundary operators and $g_{i}$ boundary control functions, $i=1,2,3$. These equations model the motion of a thermo-elastic plate, $w$ and $\theta$ being respectively the transverse deflection and temperature of the plate. We are interested in the null boundary controllability of the system. Thus we seek control functions $g_{i}$ such that $w(x, T)=w_{t}(x, T)=$ $\theta(x, T)=0$ for $x \in \Omega$.

Equations (1.1) represent a simple model for a thermo-elastic plate. More elaborate models are given by similar equations which also have nonlinear terms and a small rotational inertia term. This particular model interests us because (1.1) has smoothing properties. In fact Liu and Renardy [21] and Lasiecka and Triggiani [12], [13], [14], [16] have shown that solutions of (1.1),

2000 Mathematics Subject Classification: Primary 93B05; Secondary 35Q72, 74F05, 74K20, 74M05, 93C20.

Key words and phrases: null boundary control, Ovsyannikov theorem, thermo-elastic plate equation, heat equation. 
with various boundary conditions, are generated by analytic semigroups. Such results can also be obtained from an abstract result of Lasiecka and Triggiani [17, Theorem 3D, p. 311].

Our "balayage method" of boundary control makes use of a smoothing property of (1.1). We generate the boundary controls by solving two Cauchy problems. The first Cauchy problem involves finding a solution to (1.1) in all of $\mathbb{R}^{2}$. We shall see that such solutions are real analytic functions of $x$ and $t$ if $t>0$. An intermediate step in our method involves solving a nonhomogeneous version of (1.1). We think of the nonhomogeneous terms in the equation as being "garbage terms" which must be "swept out", a concept described by the French word "balayage". This is achieved by solving the second, non-standard Cauchy problem for which a space variable plays the role of a time variable. We use a modification of the classical Cauchy-Kowalevski theorem due to Ovsyannikov to solve this Cauchy problem. An essential ingredient is the smoothness of the solution of the first Cauchy problem.

As an example of the kind of controllability result that may be obtained by use of this method, we state the following theorem, which we prove in Section 4.

TheOREM 1.1. Assume that the controls (1.3) take the form

$$
w=g_{1}, \quad \Delta w=g_{2}, \quad \theta=g_{3} \quad \text { on } \partial \Omega \times(0, T] .
$$

Then, given $w_{0} \in H^{2}(\Omega), w_{1}, \theta_{0} \in L^{2}(\Omega)$, we can find boundary control functions $g_{i}$ such that $w(x, T)=w_{t}(x, T)=\theta(x, T)=0$ for $x \in \Omega$. Further, for all $\varepsilon>0$,

$$
g_{1} \in C^{1}\left([0, T] ; H^{3 / 2-\varepsilon}(\partial \Omega)\right), \quad g_{2}, g_{3} \in C\left([0, T] ; H^{3 / 2-\varepsilon}(\partial \Omega)\right) .
$$

The proof of Theorem 1.1 involves taking a trace on the boundary of $\Omega$ and for this some regularity must be assumed for $\Omega$. It is sufficient that $\partial \Omega$ be $C^{2}$. It is also sufficient that $\bar{\Omega}$ be the $C^{2}$ diffeomorphic image of a polyhedron $P$, the diffeomorphism being defined in an open set containing $P$. In the latter case the stated regularity of the $g_{i}$ holds for the deformed faces of the polyhedron.

The usual approaches to control of thermo-elastic plate equations make use of Carleman estimates or eigenfunction expansions. For recent work on this and related parabolic equations we refer to the papers of Lasiecka and Triggiani on exact null controllability [15]; Avalos and Lasiecka on a range of topics including boundary controllability [3], boundary reachability with variable thermal coupling [4], null controllability [5], rates of blow-up for the minimal energy function [6]; Avalos on the null controllability of von Kármán thermo-elastic plates [1]; Avalos and Cokeley on boundary and localized null controllability of structurally damped systems [2]; Cokeley on control blow-up rates [7], and Triggiani on optimal estimates of norms 
of fast controls [25]. As an example of the eigenfunction approach, which may be used for special domains, we mention the work of Lasiecka and Seidman [11]. For earlier work on thermo-elastic plates, we cite Lagnese's work on the reachability problem [10].

A rewarding feature of the balayage method is that it involves a direct calculation of the control functions. The method is a modification of ideas introduced by Littman [18]. An early form of the method was used by Littman and Markus [19] on a hybrid elastic system. A later version of the method which incorporated the Ovsyannikov theorem, a generalization of the Cauchy-Kowalevski theorem, was used by Guo and Littman [8] for the boundary controllability of a semilinear heat equation in one space dimension. More recently we modified the Ovsyannikov approach to consider boundary controllability of heat and Schrödinger equations in higher space dimensions [20] and we use this approach in the present paper.

Our method follows the following steps.

STEP 1. Extend the initial data to have compact support in $\mathbb{R}^{2}$ and find a solution of $(1.1)$ for $(x, t) \in \mathbb{R}^{2} \times(0, \infty)$. Assume for the time being that the solution functions $w$ and $\theta$ are infinitely differentiable with respect to the $t$ variable; in fact the time derivatives must satisfy Gevrey estimates. Gevrey class functions lie between the set of $C^{\infty}$ functions and the set of analytic functions; we give more details on Gevrey spaces later, but see also page 146 of [9].

STEP 2. Let $\psi(t)$, belonging to a certain Gevrey class, satisfy

$$
\psi(t)= \begin{cases}1, & t \leq T / 2 \\ 0, & t \geq T\end{cases}
$$

Such functions can be constructed explicitly. Set

$$
\begin{aligned}
& f_{1}=(w \psi)_{t t}+\Delta^{2}(w \psi)+\alpha \Delta(\theta \psi)=2 w_{t} \psi_{t}+w \psi_{t t}, \\
& f_{2}=(\theta \psi)_{t}-\beta \Delta(\theta \psi)-\alpha \Delta(w \psi)_{t}+\gamma \theta \psi=(\theta-\alpha \Delta w) \psi_{t}
\end{aligned}
$$

and note that $f_{1}(x, t)$ and $f_{2}(x, t)$ vanish for $t$ outside $[T / 2, T]$.

SteP 3. Find a solution $W, \Theta$ to the problem

$$
W_{t t}=-\Delta^{2} W-\alpha \Delta \Theta+f_{1}, \quad \Theta_{t}=\beta \Delta \Theta+\alpha \Delta W_{t}-\gamma \Theta+f_{2}
$$

for $x$ in a bounded domain $\Omega_{2}$ that contains $\bar{\Omega}$ such that $W(x, t)$ and $\Theta(x, t)$ vanish for $t$ outside $[T / 2, T]$.

STEP 4. Let $\widetilde{w}(x, t)=\psi(t) w(x, t)-W(x, t), \widetilde{\theta}(x, t)=\psi(t) \theta(x, t)-\Theta(x, t)$. Then $\widetilde{w}$ and $\widetilde{\theta}$ satisfy equations (1.1) and (1.2) and vanish for $t \geq T$. Thus the control functions may be obtained from the boundary conditions (1.3):

on $\partial \Omega \times(0, T]$.

$$
g_{i}=B_{i}(\widetilde{w}, \widetilde{\theta}), \quad i=1,2,3,
$$


There are many different ways to extend the initial data in Step 1. For the particular problem we consider here, equations (1.1)-(1.3), we desire finite energy solutions. The physical energy associated with the thermo-elastic plate is

$$
E(t)=\frac{1}{2} \int_{\Omega}\left(w_{t}^{2}+(\Delta w)^{2}+\theta^{2}\right) d x
$$

so we assume that $w_{0} \in H^{2}(\Omega)$ and $w_{1}, \theta_{0} \in L^{2}(\Omega)$. We assume that $\Omega$ is smooth enough for the existence of an extension operator $\mathcal{E}_{0}: H^{2}(\Omega) \rightarrow$ $H^{2}\left(\mathbb{R}^{2}\right)$. Such operators exist for $C^{1,1}$ domains. Furthermore, we may assume that the support of $\mathcal{E}_{0} w_{0}$ is contained in some ball $\mathcal{B}$ containing $\Omega$ (multiplying by a cut-off function equal to one on $\Omega$ would achieve this). The extension of $w_{1}, \theta_{0} \in L^{2}(\Omega)$ is simpler; we define the extensions to be equal to zero at points outside $\Omega$.

Much of this paper deals with Step 3 which involves using the Ovsyannikov theorem to find $W$ and $\Theta$.

2. Smoothing properties of the thermo-elastic plate. In this section we establish the smoothing results that we need to make use of the Ovsyannikov theorem. We give two results, the first obtained by keeping $t>0$ and analytically continuing the solution with respect to the spatial variables into $\mathbb{C}^{2}$ and the second by keeping the spatial variables real and analytically continuing with respect to $t$. The latter result corresponds to the fact that solutions are generated by an analytic semigroup.

We prefer to work with an equivalent system obtained by setting $u_{1}=w_{t}$, $u_{2}=w, u_{3}=\theta$ so that our PDE system takes the form

$$
\mathbf{u}_{t}=\left[\begin{array}{ccc}
0 & -\Delta^{2} & -\alpha \Delta \\
1 & 0 & 0 \\
\alpha \Delta & 0 & -\gamma+\beta \Delta
\end{array}\right] \mathbf{u}=\mathcal{A} \mathbf{u}, \quad \mathbf{u}(x, 0)=\mathbf{u}_{0}(x) .
$$

The Fourier representation of the solution is

$$
\mathbf{u}(x, t)=\frac{1}{2 \pi} \int_{\mathbb{R}^{2}} e^{R(\xi) t} \widehat{\mathbf{u}}_{0}(\xi) e^{i x \cdot \xi} d \xi,
$$

where

$$
R(\xi)=\left[\begin{array}{ccc}
0 & -\left(\xi_{1}^{2}+\xi_{2}^{2}\right)^{2} & \alpha\left(\xi_{1}^{2}+\xi_{2}^{2}\right) \\
1 & 0 & 0 \\
-\alpha\left(\xi_{1}^{2}+\xi_{2}^{2}\right) & 0 & -\gamma-\beta\left(\xi_{1}^{2}+\xi_{2}^{2}\right)
\end{array}\right] .
$$

It is convenient to write $R(\xi)=S(\xi)^{-1}\left(\mathbf{L}\left(\xi_{1}^{2}+\xi_{2}^{2}\right)+\mathbf{M}\right) S(\xi)$ where 
$\mathbf{L}=\left[\begin{array}{ccc}0 & -1 & -\alpha \\ 1 & 0 & 0 \\ \alpha & 0 & \beta\end{array}\right], \quad \mathbf{M}=\left[\begin{array}{ccc}0 & 0 & 0 \\ 0 & 0 & 0 \\ 0 & 0 & \gamma\end{array}\right], \quad S(\xi)=\left[\begin{array}{ccc}1 & 0 & 0 \\ 0 & -\left(\xi_{1}^{2}+\xi_{2}^{2}\right) & 0 \\ 0 & 0 & 1\end{array}\right]$

Thus we may rewrite the Fourier representation (2.2) as

$$
\mathbf{u}(x, t)=\frac{1}{2 \pi} \int_{\mathbb{R}^{2}} S(\xi)^{-1} e^{-\left(\mathbf{L}\left(\xi_{1}^{2}+\xi_{2}^{2}\right)+\mathbf{M}\right) t} S(\xi) \widehat{\mathbf{u}}_{0}(\xi) e^{i x \cdot \xi} d \xi .
$$

The convergence properties of this integral depend on the eigenvalues of $\mathbf{L}$, which has characteristic equation

$$
\lambda^{3}-\beta \lambda^{2}+\left(\alpha^{2}+1\right) \lambda-\beta=0 .
$$

The same characteristic equation appeared in the work of Liu and Renardy [21], who showed that all of the eigenvalues have positive real parts when $\alpha$ and $\beta$ are positive. The simple proof of this follows from the observation that there are no roots of the characteristic equation on the imaginary axis and therefore the signs of the real parts of the eigenvalues do not change as $\alpha$ and $\beta$ vary. It is easy to check that the eigenvalues have positive real parts when $\beta$ is small; hence this is the case for all positive $\alpha$ and $\beta$.

Choosing $\Lambda$ so that each eigenvalue $\lambda$ satisfies $\operatorname{Re} \lambda>\Lambda>0$, one easily verifies that there is some constant $C$ such that

$$
\left\|e^{-\left(\mathbf{L}\left(\xi_{1}^{2}+\xi_{2}^{2}\right)+\mathbf{M}\right) t}\right\|<C e^{-\Lambda\left(\xi_{1}^{2}+\xi_{2}^{2}\right) t}, \quad t>0 .
$$

Now, $R(\xi)=S(\xi)^{-1}\left(\mathbf{L}\left(\xi_{1}^{2}+\xi_{2}^{2}\right)+\mathbf{M}\right) S(\xi)$ is clearly bounded for small $|\xi|$. Consequently, $\exp (R(\xi) t)$ satisfies an inequality similar to (2.4),

$$
\|\exp (R(\xi) t)\|=\left\|S(\xi)^{-1} e^{-\left(\mathbf{L}\left(\xi_{1}^{2}+\xi_{2}^{2}\right)+\mathbf{M}\right) t} S(\xi)\right\|<\widetilde{C} e^{-\Lambda\left(\xi_{1}^{2}+\xi_{2}^{2}\right) t}, \quad t>0 .
$$

Hence the Fourier representation (2.2) displays $\mathbf{u}$ as an entire function of $x$ for $t>0$ (one way to see this is to verify that the power series of $(2.2)$ about $x=0$ converges for all $\left.x \in \mathbb{C}^{2}\right)$. Thus, for any compact $S \subset \mathbb{C}^{2} \times(0, \infty)$, and any $R>0$ there is a constant $C$ (related to the previous $C$ but not the same one) such that

$$
\left|D^{\mu} \mathbf{u}(x, t)\right| \leq C\left\|\mathbf{u}_{0}\right\|_{L^{2}} \frac{|\mu| !}{R^{|\mu|}}, \quad(x, t) \in S,
$$

for all $\mu$. Further, we may use the PDE (2.1), which relates time derivatives to spatial derivatives, and the previous inequality to conclude that:

Lemma 2.1. For any compact $S \subset \mathbb{C}^{2} \times(0, \infty)$ and any $\phi>0$ there is a constant $C$ such that

$$
\left|D^{\mu} \partial_{t}^{n} \mathbf{u}(x, t)\right| \leq C\left\|\mathbf{u}_{0}\right\|_{L^{2}}(|\mu|+2 n) ! \phi^{|\mu|+2 n}, \quad(x, t) \in S,
$$

for all $\mu$ and $n$. 
REMARK 2.2. One may consider the operator $\mathcal{A}$ of equation (2.1) to be an unbounded operator on the finite energy space $\mathcal{H}_{E}=L^{2}\left(\mathbb{R}^{2}\right) \times H^{2}\left(\mathbb{R}^{2}\right) \times$ $L^{2}\left(\mathbb{R}^{2}\right)$ with domain $H^{2}\left(\mathbb{R}^{2}\right) \times H^{4}\left(\mathbb{R}^{2}\right) \times H^{2}\left(\mathbb{R}^{2}\right)$. It is easy to check that $\mathcal{A}$ generates a strongly continuous semigroup $S(t)$ on $\mathcal{H}_{E}$.

For the previous result we kept $t$ real and considered $\mathbf{u}(x, t)$ for $x \in \mathbb{C}^{2}$. If instead we keep $x \in \mathbb{R}^{2}$ and allow complex $t$ we obtain a different smoothing result. Pick $0<\psi<\pi / 2$ such that each eigenvalue $\lambda$ of $\mathbf{L}$ satisfies

$$
|\arg \lambda|<\psi \text {. }
$$

This together with the boundedness of $R(\xi)$ for small $\xi$ shows that there is some constant $C$ such that

$$
\begin{array}{rlrl}
\left\|e^{-\left(\mathbf{L}\left(\xi_{1}^{2}+\xi_{2}^{2}\right)+\mathbf{M}\right) t}\right\|<C, & & |\arg t| \leq \pi / 2-\psi, \\
\left\|S(\xi)^{-1} e^{-\left(\mathbf{L}\left(\xi_{1}^{2}+\xi_{2}^{2}\right)+\mathbf{M}\right) t} S(\xi)\right\|<C, & |\arg t| \leq \pi / 2-\psi .
\end{array}
$$

This implies $\|\mathbf{u}(\cdot, t)\|_{L^{2}} \leq C\left\|\mathbf{u}_{0}\right\|_{L^{2}}$ for $|\arg t| \leq \pi / 2-\psi$. Further, we may write

$$
\left[\begin{array}{c}
u_{1}(x, t) \\
\Delta u_{2}(x, t) \\
u_{3}(x, t)
\end{array}\right]=\frac{1}{2 \pi} \int_{\mathbb{R}^{2}} e^{-\left(\mathbf{L}\left(\xi_{1}^{2}+\xi_{2}^{2}\right)+\mathbf{M}\right) t}\left[\begin{array}{c}
\widehat{u}_{10}(\xi) \\
\left(\xi_{1}^{2}+\xi_{2}^{2}\right) \widehat{u}_{20}(\xi) \\
\widehat{u}_{30}(\xi)
\end{array}\right] e^{i x \cdot \xi} d \xi,
$$

where $u_{10}, u_{20}$ and $u_{30}$ are the components of $\mathbf{u}_{0}$. So in fact we have $\|\mathbf{u}(\cdot, t)\|_{E} \leq C\left\|\mathbf{u}_{0}\right\|_{E}$ for $|\arg t| \leq \pi / 2-\psi$, where \|\|$_{E}$ denotes the usual norm for the finite energy space $L^{2} \times H^{2} \times L^{2}$. Hence:

Lemma 2.3. The semigroup $S(t)$ of Remark 2.2 is analytic in the sector $|\arg t| \leq \pi / 2-\psi$.

REMARK 2.4. Solutions of (1.1) with various boundary conditions have been shown to be generated by analytic semigroups [21], [12], [13], [14], [16]. These results are for bounded spatial domains so they are not applicable to our system. However, Lemma 2.3 also follows easily from an abstract result of Lasiecka and Triggiani [17, Theorem 3D, p. 311]. If it were not for the fact that we had already used the Fourier representation of the solution to prove Lemma 2.1 then the simplest route to Lemma 2.3 would have been via [17].

3. The solution of Step 3. We must solve

$$
W_{t t}=-\Delta^{2} W-\alpha \Delta \Theta+f_{1}, \quad \Theta_{t}=\beta \Delta \Theta+\alpha \Delta W_{t}-\gamma \Theta+f_{2}
$$

where $f_{1}=2 w_{t} \psi_{t}+w \psi_{t t}$ and $f_{2}=(\theta-\alpha \Delta w) \psi_{t}$ vanish for $t \leq T / 2$ and for $t \geq T$. We shall see that we can solve this system as a Cauchy problem with $x_{2}$ as the time variable provided that $f_{1}$ and $f_{2}$ are smooth enough. Lemma 2.1 shows that $w$ and $\theta$ are Gevrey-2 functions of $t$ (and real analytic 
in $x$ ) for $t>0$. We would also like $f_{1}$ and $f_{2}$ to have such properties. This will be the case if the cut-off function $\psi$ is chosen to belong to a "smoother" Gevrey class, such as Gevrey class 3/2. More precisely, we choose $\psi$ to satisfy $\left|\psi^{(n)}(t)\right| \leq \Gamma(3 n / 2) c R^{n}$ for all $n$, where $\Gamma$ denotes the Gamma function and $R$ and $c$ are constants that do not depend on $n$.

Definition 3.1. We introduce a compact interval $K$ such that the projection of $\bar{\Omega}$ onto the $x_{1}$ axis is contained in the interior of $K$. We will seek a solution of (3.1) in the set $K \times I \times\{t>0\}$, where $I=[0, \eta]$ and $\eta$ is chosen large enough so that Interior $(K \times I) \supset \bar{\Omega}$ (see Figure 1). We require that this solution vanish outside of $[T / 2, T]$, and assume that $\bar{\Omega} \subset\left\{x_{2}>0\right\}$.

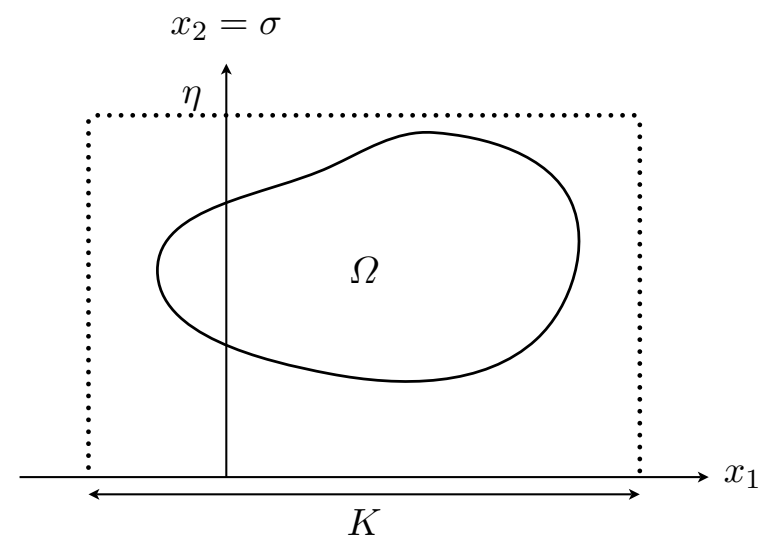

Fig. 1. The set $K$ and constant $\eta$ of Definition 3.1

3.1. Solution of equation (3.1). We use Ovsyannikov's theorem to solve (3.1). We start by setting

$$
u=\left[\begin{array}{c}
W_{t}, \\
\Delta W, \\
\Theta, \\
W
\end{array}\right], \quad v=\frac{\partial u}{\partial x_{2}}, \quad \partial_{1}=\frac{\partial}{\partial x_{1}}, \quad \partial_{t}=\frac{\partial}{\partial t}, \quad \sigma=x_{2}, \quad y=\left[\begin{array}{l}
u \\
v
\end{array}\right],
$$

and write (3.1) as the system

$$
\frac{d y}{d \sigma}=A(\sigma) y+F(\sigma),
$$

where all eight components of $F$ are zero except for $F_{6}=f_{1}+\alpha f_{2} / \beta$ and $F_{7}=-f_{2} / \beta$. The matrix entries of $A$ are

$$
A=\left[\begin{array}{cc}
\mathbf{0} & \mathbf{I} \\
\mathbf{K}+\mathbf{J} \partial_{t}-\mathbf{I} \partial_{1}^{2} & \mathbf{0}
\end{array}\right]
$$


where

$$
\mathbf{J}=\left[\begin{array}{cccc}
0 & 1 & 0 & 0 \\
-1 & \alpha^{2} / \beta & -\alpha / \beta & 0 \\
0 & -\alpha / \beta & 1 / \beta & 0 \\
0 & 0 & 0 & 0
\end{array}\right], \quad \mathbf{K}=\left[\begin{array}{cccc}
0 & 0 & 0 & 0 \\
0 & 0 & -\alpha \gamma / \beta & 0 \\
0 & 0 & \gamma / \beta & 0 \\
0 & 1 & 0 & 0
\end{array}\right]
$$

and $\mathbf{I}$ is the 4 by 4 identity matrix. We solve (3.2) subject to the initial condition $y(0)=0$ and prove the existence of solutions using the fact that $f_{1}$ and $f_{2}$ are analytic functions of $x$ and Gevrey class 2 functions of $t$.

We use the following version of the Ovsyannikov theorem.

Theorem 3.2. Let $\left(E_{s},||_{s}\right), s \in[0,1]$, be a scale of Banach spaces such that the following assumptions hold:

(1) If $s^{\prime} \leq s$ then $E_{s} \subset E_{s^{\prime}}$ and the natural injection has norm no greater than one.

(2) For $s^{\prime}<s$ and each $\sigma \in[0, \eta]$, let $A(\sigma)$ be a bounded linear mapping from $E_{s}$ into $E_{s^{\prime}}$ with operator norm no greater than $M\left(s-s^{\prime}\right)^{-1}+P$, where $M>0$ and $P \geq 0$ are constants independent of $\sigma, s$ and $s^{\prime}$. Also, $A(\sigma)$ is a continuous function of $\sigma$ in the uniform operator topology.

(3) $y_{0} \in E_{1}$ and $F \in C\left([0, \eta] ; E_{1}\right)$.

Then the problem

$$
\frac{d y}{d \sigma}=A(\sigma) y+F(\sigma), \quad y(0)=y_{0},
$$

has a unique solution belonging to $C^{1}\left(\left[0, \delta_{0}(1-s)\right] ; E_{s}\right)$ for each $s \in[0,1]$, where $\delta_{0}=\min \left(\eta,(M e)^{-1}\right)$.

REMARK 3.3. This theorem differs slightly from the classical Ovsyannikov theorem found in Trèves [24]. In the stated form of the theorem the constant $M$, which determines the length of the interval in which the solution exists, does not depend on the constant $\gamma$ of (1.1).

We omit the proof of the theorem, which is given in [23] and is not difficult to obtain by modifying the proof of the classical theorem given in $[24]$.

In the remainder of this section, we define the spaces $E_{s}$ and verify that our problem (3.2), (3.3) satisfies conditions (3.2) and (3.2) of Theorem 3.2. Verification of condition (3.2) will follow from the smoothing property, Lemma 2.1.

Definition 3.4. Let $K$ be a compact interval and let $T>0, \phi_{0}>0$, and $0<\varrho<1$. For $s \in[0,1]$ and $p \geq 0$, we define $B_{s, p}$ to be the set of all 
$f \in C^{\infty}(K \times \mathbb{R})$ such that $f(x, t)=0$ for $t$ outside $[T / 2, T]$ and

$$
|f|_{s, p} \equiv|f|_{s, p}^{K}=\sup _{n, \mu} \max _{x, t} \frac{\left|\frac{\partial^{n+\mu} f}{\partial t^{n} \partial x^{\mu}}\right|}{\left(\phi_{0} \varrho^{s}\right)^{2(n+p)+\mu+p}(2 n+\mu) !(2 n+\mu+1)^{2 p}}<\infty,
$$

where the supremum is taken over all integers $n, \mu \geq 0$.

Remark 3.5. The set $K$ appearing in Definition 3.4 is identical to the set introduced in Definition 3.1, and the interval $[0, \eta]$ of condition (3) of Theorem 3.2 is identical to the interval appearing in Definition 3.1.

REMARK 3.6. Members of $B_{s, p}$ are functions of $x_{1}$ and $t$ and the solution $y$ of (3.2) is a function of $x_{2}=\sigma$ with components taking values in certain $B_{s, p}$ spaces. Functions belonging to $B_{s, p}$ satisfy Gevrey-2 bounds for their time derivatives and analyticity bounds for their space derivatives.

It is easily seen that ||$_{s, p}$ is a norm on $B_{s, p}$ which makes $B_{s, p}$ a Banach space. For fixed $p$ and with $s$ ranging over $[0,1]$ the spaces $B_{s, p}$ form a scale of Banach spaces in the sense that if $s^{\prime} \leq s$ then $B_{s, p} \subset B_{s^{\prime}, p}$ and the natural injection has norm no greater than one.

Definition 3.7. For $s \in[0,1]$ we define $E_{s}=\left(B_{s, 0}\right)^{4} \times\left(B_{s, 1 / 2}\right)^{4}$.

We need to consider the norm of $A$ as a mapping from $E_{s}$ to $E_{s^{\prime}}$ with $s^{\prime}<s$. The expression for $A$ given in (3.3) shows that this is the sum of the norms of two other maps:

- the natural injection from $\left(B_{s, 1 / 2}\right)^{4}$ into $\left(B_{s^{\prime}, 0}\right)^{4}$,

- $\mathbf{K}+\mathbf{J} \partial_{t}-\mathbf{I} \partial_{1}^{2}$ from $\left(B_{s, 0}\right)^{4}$ into $\left(B_{s^{\prime}, 1 / 2}\right)^{4}$.

Luckily we have already considered similar mappings in [20]. With the constant $\theta_{0}$ of Part 1 of Lemma 2.8 in [20] given by $\theta_{0}=\phi_{0}^{2}$, the lemma shows that the natural injection from $B_{s, 1 / 2}$ into $B_{s^{\prime}, 0}$ has norm no greater than

$$
\frac{\phi_{0}^{3 / 2}}{\left(s-s^{\prime}\right) e \log (1 / \varrho)} \text {. }
$$

Also, by Part 2 of [20, Lemma 2.8], $\partial_{1}^{2}$ and $\partial_{t}$, regarded as mappings from $B_{s, 0}$ into $B_{s^{\prime}, 1 / 2}$, have norms no greater than

$$
\frac{\phi_{0}^{1 / 2}}{\left(s-s^{\prime}\right) e \log (1 / \varrho)} \text {. }
$$

It is easy to check that the norm of $\mathbf{K}$, regarded as a mapping from $B_{s, 0}$ into $B_{s^{\prime}, 1 / 2}$, has a norm which is a bounded function of $s$ and $s^{\prime}$. This mapping contributes only to the constant $P$ of the Ovsyannikov theorem. 
The constant $M$ of that theorem thus satisfies

$$
M \leq C(\alpha, \beta) \frac{\phi_{0}^{3 / 2}+\phi_{0}^{1 / 2}}{\log (1 / \varrho)},
$$

where $C(\alpha, \beta)$ denotes a constant that depends on the constants $\alpha$ and $\beta$ of the thermo-elastic PDEs.

The interval of existence of the Ovsyannikov solution is the whole interval $[0, \eta]$ provided that $M e<1 / \eta$. Fix any $\varrho \in(0,1)$. It follows that the interval of existence is all of $[0, \eta]$ if $\phi_{0}$ is chosen so small that

$$
e C(\alpha, \beta) \frac{\phi_{0}^{3 / 2}+\phi_{0}^{1 / 2}}{\log (1 / \varrho)}<1 / \eta
$$

Finally, we must check that our function $F$, defined by (3.2), satisfies condition (3) of Theorem 3.2, i.e. $F \in C\left([0, \eta] ; E_{1}\right)$. But, by Definition 3.7, this is equivalent to the functions $\sigma \mapsto f_{1}(\cdot, \sigma, \cdot)$ and $\sigma \mapsto f_{2}(\cdot, \sigma, \cdot)$ of equation (3.3) belonging to $C\left([0, \eta] ;\left(B_{1,1 / 2}\right)^{4}\right)$. Recall that $f_{1}=2 w_{t} \psi_{t}+w \psi_{t t}$ and $f_{2}=(\theta-\alpha \Delta w) \psi_{t}$, where $\psi$ is a Gevrey class $3 / 2$ function and $w, \theta$ is the solution of the Cauchy problem of Section 1 . This allows us to make use of Lemma 2.1. In fact, if we choose the constant $\phi$ of Lemma 2.1 to satisfy $\phi<\varrho \phi_{0}$ then it is easy to see that $F$ has the desired regularity.

4. Massaging the method. Now that the construction of the solution to Step 3 in the introduction is done, one could construct the boundary control functions as in Step 4. The boundary control functions are found by taking the trace of a function which is $C^{\infty}$ for $t>0$. Hence the control function is as smooth as the boundary operators $\mathbf{B}_{i}$ and boundary $\partial \Omega$ allow, for $t>0$. However, this smoothness is not valid at $t=0$. In this section we see how to "massage the method" in order to improve the smoothness at $t=0$.

DeFinition 4.1. For $t \geq 0$, let $\Phi(t): L^{2}(\Omega) \times H^{2}(\Omega) \times L^{2}(\Omega) \rightarrow L^{2}(\Omega) \times$ $H^{2}(\Omega) \times L^{2}(\Omega)$ denote the solution operator $\left(w_{1}, w_{0}, \theta_{0}\right) \mapsto\left(w_{t}(\cdot, t), w(\cdot, t)\right.$, $\theta(\cdot, t))$ obtained by following Steps $1-4$ as outlined in the Introduction.

The operator $\Phi(t)$ is $C^{\infty}$ in the uniform operator topology for $t>0$, but at $t=0$ all that we can say is that $\Phi(t)$ is strongly continuous (and this is because $\Phi(t)$ coincides with the $C_{0}$ semigroup $S(t)$ of Remark 2.2 for $0 \leq t \leq T / 2)$. Thus the smoothness of the boundary controls is lost at $t=0$.

We illustrate our massaging approach for the case of the controls taking the form

$$
w=g_{1}, \quad \Delta w=g_{2}, \quad \theta=g_{3} \quad \text { on } \partial \Omega \times(0, T] .
$$

In this case, we proceed as follows: 
(1) Solve the mixed problem consisting of equation (2.1) in $\Omega \times(0, \infty)$ with the homogeneous boundary conditions

$$
u_{1}=0, \quad \Delta u_{2}=u_{2}=0, \quad u_{3}=0 \quad \text { on } \partial \Omega \times(0, T] .
$$

We denote the solution of this problem by $\mathbf{u}_{1}(x, t)$.

(2) Let $\psi(t)$ be the same cut-off function introduced in Section 3 and let $\mathbf{u}_{2}(x, t)=\mathbf{u}_{1}(x, t) \psi(t)$. Thus $\mathbf{u}_{2}$ satisfies the initial condition, has zero Dirichlet data and vanishes for $t>T$. But the PDE satisfied by $\mathbf{u}_{2}$ is nonhomogeneous:

$$
\frac{\partial \mathbf{u}_{2}}{\partial t}(x, t)=\mathcal{A} \mathbf{u}_{2}(x, t)+\mathbf{G}(x, t),
$$

where $\mathbf{G}(x, t)=\mathbf{u}_{1}(x, t) \psi^{\prime}(t)$.

(3) Use Duhamel's principle to solve the same nonhomogeneous PDE:

$$
\mathbf{u}_{3}(\cdot, t)=\int_{0}^{t} \Phi(t-\tau) \mathbf{G}(\cdot, \tau) d \tau .
$$

Now $\mathbf{G}(x, t)$ vanishes for $t$ outside $[T / 2, T]$ so $U(x, t)=\mathbf{u}_{2}(x, t)-\mathbf{u}_{3}(x, t)$ satisfies equation (2.1) and vanishes for $t \geq 2 T$. The boundary controls are given by

$$
g_{1 t}(x, t)=u_{31}(x, t), \quad g_{2}(x, t)=\Delta u_{32}(x, t), \quad g_{3}(x, t)=u_{33}(x, t), \quad x \in \partial \Omega,
$$

where $\mathbf{u}_{3}=\left(u_{31}, u_{32}, u_{33}\right)$. Thus we need to consider the regularity of $\mathbf{u}_{3}$.

The operator $\Phi(t)$ coincides with the analytic semigroup $S(t)$ of Lemma 2.3 for $0 \leq t \leq T / 2$. The domain of the infinitesimal generator of this semigroup is $\mathcal{D}=H^{2}\left(\mathbb{R}^{2}\right) \times H^{4}\left(\mathbb{R}^{2}\right) \times H^{2}\left(\mathbb{R}^{2}\right)$. A standard result for analytic semigroups (see, for example, Pazy [22]) tells us that, for $t>0, S(t)$ maps $\mathcal{H}_{E}=L^{2}\left(\mathbb{R}^{2}\right) \times H^{2}\left(\mathbb{R}^{2}\right) \times L^{2}\left(\mathbb{R}^{2}\right)$ into $\mathcal{D}$ and the norm of the mapping is bounded by $C / t$ for some constant $C$. Hence $\Phi(t): L^{2}(\Omega) \times H^{2}(\Omega) \times L^{2}(\Omega) \rightarrow$ $H^{2}(\Omega) \times H^{4}(\Omega) \times H^{2}(\Omega)$, with norm bounded by $C / t$ for some constant $C$. By interpolation, $\Phi(t): L^{2}(\Omega) \times H^{2}(\Omega) \times L^{2}(\Omega) \rightarrow H^{2 s}(\Omega) \times H^{2 s+2}(\Omega) \times$ $H^{2 s}(\Omega), 0 \leq s \leq 1$, with norm bounded by $C / t^{s}$. Hence

$$
\begin{aligned}
\left\|\mathbf{u}_{3}(\cdot, t)\right\|_{H^{2 s} \times H^{2 s+2} \times H^{2 s}} & \leq C\left\|\mathbf{u}_{0}\right\|_{L^{2} \times H^{2} \times L^{2}} \max \left(\left|\psi^{\prime}\right|\right) \int_{0}^{t}(t-\tau)^{-s} d \tau \\
& \leq C^{\prime}\left\|\mathbf{u}_{0}\right\|_{L^{2} \times H^{2} \times L^{2}}
\end{aligned}
$$

Thus, assuming that the trace theorem holds for $\partial \Omega$, we find that the boundary control functions satisfy

$$
g_{1 t}, g_{2}, g_{3} \in L^{\infty}\left([0, \infty) ; H^{3 / 2-\varepsilon}(\partial \Omega)\right), \quad \varepsilon>0 .
$$

The strong continuity of the semigroup gives us a little more:

$$
g_{1 t}, g_{2}, g_{3} \in C\left([0, \infty) ; H^{3 / 2-\varepsilon}(\partial \Omega)\right), \quad \varepsilon>0 .
$$


We finally note that this yields a proof of Theorem 1.1, apart from the fact that we have a control time $2 T$ instead of $T$. But $T>0$ is arbitrary, so Theorem 1.1 holds as stated.

Other kinds of boundary control operators may be treated similarly.

5. Concluding remarks. Perhaps the most important aspect of our method is that it yields control functions directly. Essentially they are obtained by solving two Cauchy problems.

The control functions that we obtain by this method are defined on the whole boundary but there are ways to include fixed boundary conditions on parts of the boundary. Suppose, for example, that a thermo-elastic plate is clamped along a straight section of the boundary. In this case the Ovsyannikov solution could be computed with zero initial values on this part of the boundary, thereby preserving the clamped boundary conditions.

We should note that nonlinear PDEs may be treated as well and we cite for this the work by Guo and Littman [8] on the boundary controllability of a semilinear heat equation in one space dimension.

\section{References}

[1] G. Avalos, Null controllability of von Kármán thermo-elastic plates under the clamped or free mechanical boundary conditions, J. Math. Anal. Appl. 318 (2006), 410-432.

[2] G. Avalos and P. Cokeley, Boundary and localized null controllability of structurally damped elastic systems, in: Control Methods in PDE-Dynamical Systems, Contemp. Math. 426, Amer. Math. Soc., Providence, RI, 2007, 57-78.

[3] G. Avalos and I. Lasiecka, Boundary controllability of thermo-elastic plates via the free boundary conditions, SIAM J. Control Optim. 38 (2000), 337-383.

[4] - - - Exact-approximate boundary reachability of thermo-elastic plates under variable thermal coupling, Inverse Problems 16 (2000), 979-996, .

[5] - - - , The null controllability of thermo-elastic plates and singularity of the associated minimal energy function, J. Math. Anal. Appl. 294 (2004), 34-61.

[6] - - - Asymptotic rates of blowup for the minimal energy function for the null controllability of thermo-elastic plates: the free case, in: Control Theory of Partial Differential Equations, Lecture Notes in Pure Appl. Math. 242, Chapman \& Hall/CRC, Boca Raton, FL, 2005, 1-27.

[7] P. Cokeley, Localized null controllability and corresponding minimal norm control blowup rates of thermo-elastic systems, J. Math. Anal. Appl. 336 (2007), 140-155.

[8] Y.-J. L. Guo and W. Littman, Null boundary controllability for semilinear heat equations, Appl. Math. Optim. 32 (1995), 281-316.

[9] L. Hörmander, Linear Partial Differential Operators, Grundlehren Math. Wiss. 116, Springer, New York, 1963.

[10] J. E. Lagnese, The reachability problem for thermo-elastic plates, Arch. Ration. Mech. Anal. 112 (1990), 223-267.

[11] I. Lasiecka and T. I. Seidman, Blowup estimates for observability of a thermo-elastic system, Asymptotic Anal. 50 (2006), 93-120. 
[12] I. Lasiecka and R. Triggiani, Analyticity, and lack thereof, of thermo-elastic semigroups, in: Control and Partial Differential Equations (Marseille-Luminy, 1997), ESAIM Proc. 4, Soc. Math. Appl. Indust., Paris, 1998, 199-222.

[13] -, -, Analyticity of thermo-elastic semigroups with coupled hinged/Neumann B.C, Abstr. Appl. Anal. 3 (1998), 153-169.

[14] - - - Analyticity of thermo-elastic semigroups with free boundary conditions, Ann. Scuola Norm. Sup. Pisa Cl. Sci. (4) 27 (1998), 457-482.

[15] - - - Exact null controllability of structurally damped and thermo-elastic parabolic models, Atti Accad. Naz. Lincei Cl. Sci. Fis. Mat. Natur. Rend. Lincei (9) Mat. Appl. 9 (1998), 43-69.

[16] - - - Two direct proofs on the analyticity of the s.c. semigroup arising in abstract thermo-elastic equations, Adv. Differential Equations 3 (1998), 387-416.

[17] - - - Control Theory for Partial Differential Equations: Continuous and Approximation Theories. I. Abstract Parabolic Systems, Encyclopedia Math. Appl. 74, Cambridge Univ. Press, Cambridge, 2000.

[18] W. Littman, Boundary control theory for beams and plates, in: Proc. 24th Conf. on Decision and Control (Ft. Lauderdale, FL, 1985), IEEE Computer Soc., Los Angeles, CA, 1985, 2007-2009.

[19] W. Littman and L. Markus, Exact boundary controllability of a hybrid system of elasticity, Arch. Ration. Mech. Anal. 103 (1988), 193-236.

[20] W. Littman and S. Taylor, The heat and Schrödinger equations: boundary control with one shot, in: Control Methods in PDE-Dynamical Systems, Contemp. Math. 426, Amer. Math. Soc., Providence, RI, 2007, 293-305.

[21] Z.-Y. Liu and M. Renardy, A note on the equations of a thermoelastic plate, Appl. Math. Lett. 8 (1995), 1-6.

[22] A. Pazy, Semigroups of Linear Operators and Applications to Partial Differential Equations, Appl. Math. Sci. 44, Springer, New York, 1983.

[23] S. W. Taylor, Gevrey regularity of solutions of evolution equations and boundary controllability, PhD thesis, Univ. of Minnesota, 1989.

[24] F. Trèves, Ovsyannikov Theorem and Hyperdifferential Operators, Notas Mat. 46, Inst. Mat. Pura Apl., Conselho Nacional de Pesquisas, Rio de Janeiro, 1968.

[25] R. Triggiani, Optimal estimates of norms of fast controls in exact null controllability of two non-classical abstract parabolic systems, Adv. Differential Equations 8 (2003), 189-229.

School of Mathematics

University of Minnesota

Minneapolis, MN 55455, U.S.A.

E-mail: littman@math.umn.edu
Mathematics Department The University of Auckland Private Bag 92019

Auckland, New Zealand E-mail: s.taylor@auckland.ac.nz

Received on 10.4.2008;

revised version on 10.9.2008 
